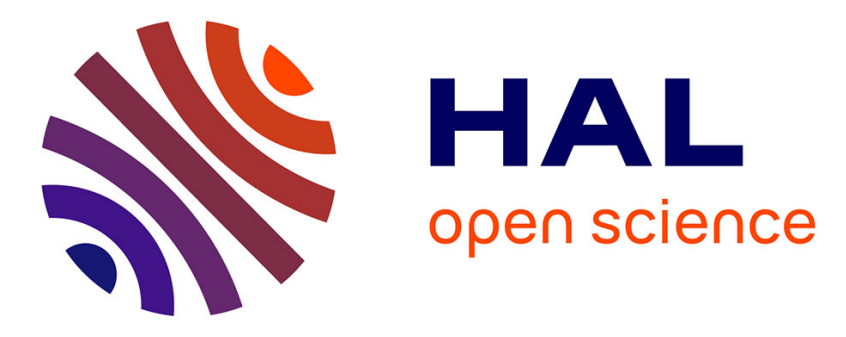

\title{
Carbon Nanotubes Induced Gelation of Unmodified Hyaluronic Acid
}

Camilo Zamora-Ledezma, Lionel Buisson, Simon E. Moulton, Gordon

Wallace, Cecile Zakri, Christophe Blanc, Eric Anglaret, Philippe Poulin

\section{- To cite this version:}

Camilo Zamora-Ledezma, Lionel Buisson, Simon E. Moulton, Gordon Wallace, Cecile Zakri, et al.. Carbon Nanotubes Induced Gelation of Unmodified Hyaluronic Acid. Langmuir, 2013, 29 (32), pp.10247-10253. 10.1021/la4016492 . hal-00870751

\section{HAL Id: hal-00870751 https://hal.science/hal-00870751}

Submitted on 29 Sep 2017

HAL is a multi-disciplinary open access archive for the deposit and dissemination of scientific research documents, whether they are published or not. The documents may come from teaching and research institutions in France or abroad, or from public or private research centers.
L'archive ouverte pluridisciplinaire HAL, est destinée au dépôt et à la diffusion de documents scientifiques de niveau recherche, publiés ou non, émanant des établissements d'enseignement et de recherche français ou étrangers, des laboratoires publics ou privés. 


\title{
Carbon Nanotubes Induced Gelation of Unmodified Hyaluronic Acid
}

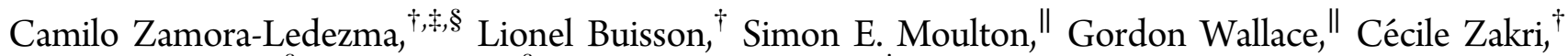 \\ Christophe Blanc, ${ }^{\S}$ Eric Anglaret, ${ }^{\S}$ and Philippe Poulin ${ }^{*, \dagger}$
}
${ }^{\dagger}$ Centre de Recherche Paul-Pascal, Université de Bordeaux - CNRS, 115 Avenue Schweitzer, 33600 Pessac, France
${ }^{\ddagger}$ Laboratorio de Física de la Materia Condensada, Centro de Física, Instituto Venezolano de Investigaciones Científícas, Altos de Pipe, 1204 Caracas, Venezuela
${ }^{\S}$ Laboratoire Charles Coulomb UMR 5221, Université Montpellier II, place Eugène Bataillon, 34095 Montpellier Cedex 5, France
"ARC Centre of Excellence for Electromaterials Science, Intelligent Polymer Research Institute, University of Wollongong, New South Wales, Australia

\begin{abstract}
This work reports an experimental study of the kinetics and mechanisms of gelation of carbon nanotubes (CNTs)-hyaluronic acid (HA) mixtures. These materials are of great interest as functional biogels for future medical applications and tissue engineering. We show that CNTs can induce the gelation of noncovalently modified HA in water. This gelation is associated with a dynamical arrest of a liquid crystal phase separation, as shown by small-angle light scattering and polarized optical microscopy. This phenomenon is reminiscent of arrested phase separations in other colloidal systems in the presence of attractive interactions. The gelation
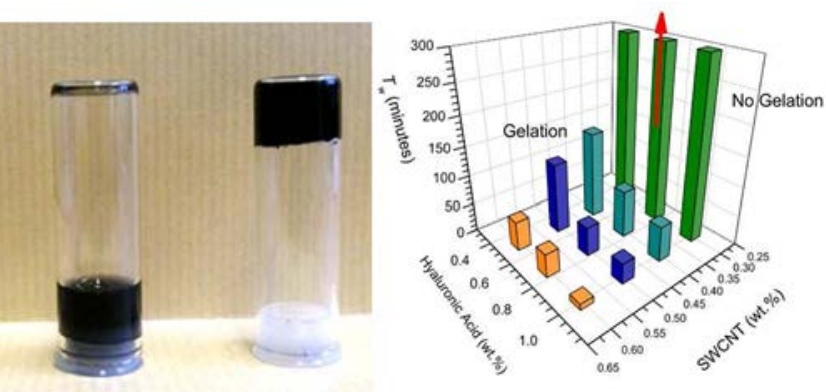
time is found to strongly vary with the concentrations of both HA and CNTs. Near-infrared photoluminescence reveals that the CNTs remain individualized both in fluid and in gel states. It is concluded that the attractive forces interplay are likely weak depletion interactions and not strong van der Waals interactions which could promote CNT rebundling, as observed in other biopolymer-CNT mixtures. The present results clarify the remarkable efficiency of CNT at inducing the gelation of HA, by considering that CNTs easily phase separate as liquid crystals because of their giant aspect ratio.
\end{abstract}

\section{INTRODUCTION}

Hyaluronic acid (HA) is a common component of synovial fluid extracellular matrix. This natural polysaccharide is of great interest for medical and cosmetic applications. ${ }^{1}$ It is particularly promising for uses in regenerative medicine, drug delivery systems, and tissue engineering. ${ }^{1-5}$ In most applications HA has to be used in a gel state. Unfortunately, raw HA in aqueous media does increase the viscosity of the solution but does not form elastic gels. ${ }^{6-9}$ Consequently extensive research work has been devoted over the last years to develop chemical modifications of HA and covalent cross-linkers. ${ }^{1,2,10,11}$ These chemical modifications can lead to the formation of gels. Other approaches such as the addition of metal ions or the addition of mineralizing species have also been shown to be efficient at forming HA-based hydrogels. ${ }^{9,12}$ On the other hand, it was recently reported that the addition of carbon nanotubes (CNTs) could also lead to the formation of HA gels. ${ }^{13-15}$ These gels are achieved at relatively low concentrations without any covalent modifications of the polymer. HA-CNT composites have also been investigated as biofibers ${ }^{16}$ or as electrode materials for bioelectrochemical applications. ${ }^{17-21}$ The new HA-CNT biogels have already demonstrated promising properties and biofunctionalities. They are biocompatible, electrically conductive, and can serve as scaffolds with electrically stimulated delivery of bioactive molecules such as neurotrophins. ${ }^{15}$ Nevertheless, in spite of their great interest, the mechanisms and kinetics of gelation of HA-CNT systems are still poorly known. These are key features to develop future applications and advance our understanding on the interactions between biomolecules and carbon nanotubes. In particular, knowing the time needed to form a gel is of critical importance to process the material in a desired shape. Gelation of HACNT mixtures could involve several effects. CNTs can be viewed as stiff, long hydrophobic rods. As produced single walled CNTs (SWCNTs) are generally in bundles. Partial or full debundling can be achieved by supplying mechanical energy via sonication, high shear mixing, or ball milling. Stabilization of the nanotubes is then necessary to avoid rebundling in response to van der Waals interactions. It was observed that HA molecules could stabilize SWCNTs in aqueous media at low concentration. Dilute dispersions remain fluid and isotropic. Nevertheless, this observation does not mean that the nanotubes are actually individualized. They could still be homogeneously dispersed as small bundles. The mixtures form gels when the concentration of $\mathrm{HA}$ and CNT are increased. ${ }^{13}$ The gels exhibit a liquid crystalline texture with 
small monodomains of the nematic phase. It was suggested that the phase separation and associated gelation were promoted by so-called depletion interactions. ${ }^{13,22,23}$ Nevertheless, it is not clear if other effects could be involved such as van der Waals interactions, which could induce aggregation and rebundling of the CNTs. ${ }^{13,24}$ It is also possible that CNTs are not efficiently unbundled at high concentration as observed in aqueous mixtures of CNTs and other biomacromolecules. ${ }^{25}$ Lastly, one can also consider that CNT and HA molecules form complexes with an extreme persistence length, increasing thereby the viscosity of the materials. Indeed the persistence length of $\mathrm{CNTs}^{26-28}(>10 \mu \mathrm{m})$ exceeds by far the persistence of HA molecules $^{8}(\sim 8 \mathrm{~nm})$.

We report in this work an experimental study of HA-CNT biogels using polarized optical microscopy (POM), small angle light scattering (SALS), and coupled photoluminescence/ Raman spectroscopies (PL/RS). These complementary techniques provide new insights into the gelation mechanisms and kinetics of formation of HA-CNT biogels. POM allows the gelation to be associated with the formation of a phase separated network of nematic phase. SALS is a nondestructive and particularly suitable technique to study phase separation phenomena and colloidal aggregation. ${ }^{29-34}$ Indeed, these phenomena result in the formation of large structures with characteristic sizes of several micrometers. They are therefore associated to an increase of scattered light at small angles. ${ }^{29-34}$ It is presently observed that the intensity of scattered light at small angles increases after a certain time and then levels off. This behavior is reminiscent of an arrested phase separation as observed in colloidal systems in the presence of attractive interactions. $^{35-39}$ We quantitatively determine from these experiments the gelation time as a function of the concentrations of HA and CNTs. This time is found to vary from a few minutes to several hours, depending on the concentrations of the materials. PL provides insights on the behavior of the nanotubes at a microscopic scale. SWCNTs can exhibit metallic or semiconductor behavior depending on their structure. ${ }^{40,41}$ Semiconducting SWCNT can absorb light over a broad spectral range in the UV-visible-IR region and emit light in the range of $1000-1800 \mathrm{~nm}$, falling in the near-infrared spectral region. ${ }^{42}$ This property which is called near-infrared photoluminescence (NIR PL) is particularly promising for bioimaging applications because it offers minimal interfering fluorescence from biological materials and superior tissue penetration depth. Nevertheless, as-produced SWCNTs do not exhibit PL because contacts between metallic and semiconducting tubes in the bundles quench the PL signal. ${ }^{42}$ Therefore, PL is observed only when the nanotubes are individualized. PL of HA stabilized SWCNTs is presently observed both in fluid dispersions and nematic gels. This finding confirms debundling and stabilization of individual nanotubes by HA molecules in the fluid suspensions. This suggests that gelation results from an arrested phase separation essentially induced by weak attractive depletion forces. The present findings also provide a better understanding of the electrical properties of the HA-CNT composite and biogels. Indeed, the presence of HA molecules strongly adsorbed at the interface of the CNTs can explain the large impedance of CNT-HA biofilms and electrode materials compared to other CNT-biopolymer systems. ${ }^{15}$

\section{EXPERIMENTAL SECTION}

Hyaluronic acid potassium salt from human umbilical cord $\left(\mathrm{C}_{14} \mathrm{H}_{20} \mathrm{KNO}_{11}\right)$ (CAS Number 31799-91-4) was obtained from United States Biological (Salem, MA). This commercial salt has a broad molar mass range of 500-3000 kg. It is provided under the form of a white freeze-dried powder. This material is water-soluble and provides solutions with a $\mathrm{pH}$ of about 7 in deionized water. RawSWCNTs bundles powder was obtained from Unidym (Sunnyvale, CA, batch number R0556). These nanotubes are synthesized by a Chemical Vapor Deposition process called the HiPco process (highpressure carbon monoxide). ${ }^{43}$ As-produced materials contain a large weight fraction, of about $25 \mathrm{wt} \%$, of iron nanoparticles.$^{44}$ An acidbased purification process is used to remove the metal nanoparticles. ${ }^{45}$ The SWCNT powder is first oxidized in an air atmosphere at $200{ }^{\circ} \mathrm{C}$ overnight and then mixed and stirred in a concentrated aqueous solution of $\mathrm{HCl}(1 \mathrm{~N})$ at room temperature. This treatment does not oxidize the nanotubes and therefore does not downgrade the structure and properties of the nanotubes. Indeed, the oxidation process in air at $200{ }^{\circ} \mathrm{C}$ affects essentially iron nanoparticles to produce iron oxide which is soluble in acidic media. The nanotubes are subsequently filtered and rinsed with abundant water to stabilize a neutral $\mathrm{pH}$. The amount of iron content after purification is about 4 wt \%, as determined by thermal gravimetric analysis. Remaining iron is essentially encapsulated in the nanotubes. This remaining iron is therefore not expected to affect the behavior of the CNTs and HA molecules in solution. The nanotubes are not dried after purification but are instead kept under the form of a wet cake that contains $7.8 \mathrm{wt}$ $\%$ of purified SWCNTs. Wet cakes can more easily be redispersed in an aqueous media than materials that have been dried. It is believed that drying leads to the formation and compaction of aggregates that cannot be easily disrupted afterward. The HA polymer was directly dissolved into water overnight at $4{ }^{\circ} \mathrm{C}$ and then shaken during $60 \mathrm{~min}$ at room temperature prior to use. The obtained HA solutions are transparent, homogeneous, and fluid. Nevertheless, their viscosity is dependent on the HA concentration as already reported in the literature. ${ }^{6-9}$ We stress that even at long times of several days, these solutions do not form gels and remain perfectly fluid. Lastly, HA solutions are mixed with the purified SWCNTs to prepare $1 \mathrm{~mL}$ of suspension. The suspension is sonicated with a Branson Sonifier S$250 \mathrm{~A}$ equipped with a $13 \mathrm{~mm}$ step disruptor horn and a $3 \mathrm{~mm}$ tapered microtip, operating at a $20 \mathrm{kHz}$ frequency. During sonication, the vial with the mixture is dipped into cold water to avoid overheating. Dark, homogeneous, and stable nanotube suspensions are obtained after this step. The average length of the raw CNTs exceeds several micrometers. ${ }^{46}$ Nevertheless, the CNTs are shortened during the sonication process. ${ }^{47,48}$ In the present case, while the exact size of the nanotubes has not been determined, the average length is expected to lie between 500 and $1000 \mathrm{~nm}$, considering related experiments on similar materials. ${ }^{46}$ The aspect ratio of the CNTs as defined by the ratio of the length over the diameter is therefore expected to be above 500. At each step of the dispersion and gel formation, dispersion at the nanoscale is investigated using coupled PL/RS characterizations. The experiments are carried out with a Bruker RFS100 FT Raman spectrometer, equipped with a nitrogen-cooled germanium detector. The excitation laser line is a Nd:YAG emitting at $1.16 \mathrm{eV}$. This energy allows the observation on the same spectra of both the PL signal and the Stokes Raman signal in the range of 100 and $3200 \mathrm{~cm}^{-1}$, corresponding to a wavelength range of about $1100-1600 \mathrm{~nm}$ (an energy range of about $1.15-0.77 \mathrm{eV}$ ).

The sample homogeneity and texture at the micrometer scale, as well as the birefringence, are assessed by optical microscopy. The kinetics of gelation are investigated in the reciprocal space by SALS. A homemade setup was designed and used for the present study. The SALS setup is sketched in Figure 1. The light source is a $1.00 \mathrm{~mW}$ $\mathrm{He}-\mathrm{Ne}$ laser, linearly polarized, with $\lambda=632.8 \mathrm{~nm}$ and a beam size at $1 / e^{2}$ of $0.55 \mathrm{~mm}$ and a full angle divergence of $1.5 \mathrm{mrad}$. A variable metallic neutral density filter can reduce the beam power down to a few $\mu \mathrm{W}$, as required for the present measurements. A polarizer sets the polarization of the electrical field in the vertical direction. The incident 


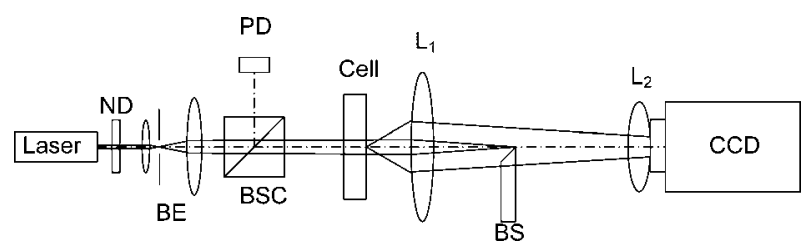

Figure 1. Schematic of the small-angle light scattering instrument. ND, variable neutral density; $\mathrm{BE}$, beam expander; $\mathrm{BSC}$, beam splitter cube; PD, photodiode; L1, collecting lens; BS, beam stop; and L2, camera lens.

beam is spatially filtered, expanded to a $1 / e^{2}$ diameter of $5 \mathrm{~mm}$. Half of the incident beam is forwarded onto a photodiode with a beam splitter cube. Then, the beam lights the sample which is contained in a $10 \mu \mathrm{m}$ thick quartz cell. Quartz cells are first carefully cleaned with water, acetone, and ultrapure isopropyl alcohol and then wiped with an optical paper. Remaining dust is removed by using compressed dry-air. A drop of the HA-CNT suspension is placed on a quartz substrate, and the cell is formed by carefully placing another quartz substrate on top of it. The cell is then sealed with Parafilm wax paper to avoid water evaporation. It is placed in the sample holder and slightly tilted with respect to the optical axis to avoid beam reflections onto the CCD camera sensor. The detection optics which collect the scattered light is similar to that described by Ferri et al. ${ }^{49}$ The scattered and transmitted beams are collected by a lens $L_{1}$ (Achromat $f=75 \mathrm{~mm}$, diameter $=50$ $\mathrm{mm}$ ). In the focal plane of $L_{1}$, a drill tip cut and polished at $45^{\circ}$ stops the unscattered light to preserve the CCD sensor. The CCD objective $L_{2}$ (Nikon, $50 \mathrm{~mm}, f / 1.2$ ) conjugates the plane of the CCD sensor and the focal plane of the collecting lens $L_{1}$, with a magnification ratio of 9 . Each CCD pixel corresponds to a different scattering wave vector $q$, with $q=4 \pi \lambda^{-1} \sin (\theta / 2)$, and where $\theta$ is the scattering angle. The light scattered at the same wave vector $q$, mapped on the CCD sensor into concentric rings centered on the optical axis, is averaged over rings of pixels. In our experimental setup, the angles for which reliable data can be collected range from $\theta=0.1^{\circ}$ to $\theta=6.3^{\circ}$; they correspond to wave vectors in the range of $0.018 \mu \mathrm{m}^{-1}<q<1.1 \mu \mathrm{m}^{-1}$.

The minimum angle is limited by the size of the drill tip that stops the transmitted beam, while the maximum angle is limited by the size of the lens $L_{1}$. The CCD camera is a 12-bit digital camera (Pulnix RM4200GE) with a $2046 \times 2046$ pixels sensor, each pixel area being $7.4 \times$ $7.4 \mu \mathrm{m}^{2}$. The digitized images are acquired by a PC. In a typical experiment, a series of a few frames is collected, each at various exposure times. Subtraction of stray light contribution is performed by acquiring the image of the cell filled by the solvent taken with the same exposure time. Then, light intensity is averaged over rings on the CDD sensor and corrected by the transmittance of the sample a factor. The corrected scattered intensity is finally plotted as a function of the $q$ wave vector for different times.

\section{RESULTS AND DISCUSSION}

A typical macroscopic photograph of a 0.5 wt \% $\mathrm{HA}$ aqueous solution is shown in Figure 2a. The solution is transparent and fluid. A suspension comprising of $0.3 \mathrm{wt} \%$ of SWCNTs and 0.25 wt \% of HA is shown in Figure $2 b$. This system remains fluid even after several days with no particular texture observed by optical microscopy. By contrast, systems that contain more $\mathrm{HA}$ and CNTs form gels as shown in Figure 2c. In addition, these gels exhibit a texture that can be visualized by POM. Typical optical micrographs with and without crossed polarizers are shown in Figure 2 (panels $\mathrm{d}$ and e). The gels do not result from the random aggregation or jamming of the CNTs or from a large increase of viscosity due to the formation of complexes with long persistence length. This phenomenon would lead to a gradual and continuous increase of the viscosity but not to the sudden apparition of elastic gels above critical thresholds of HA and CNT concentrations. Here, small birefringent grains reflect

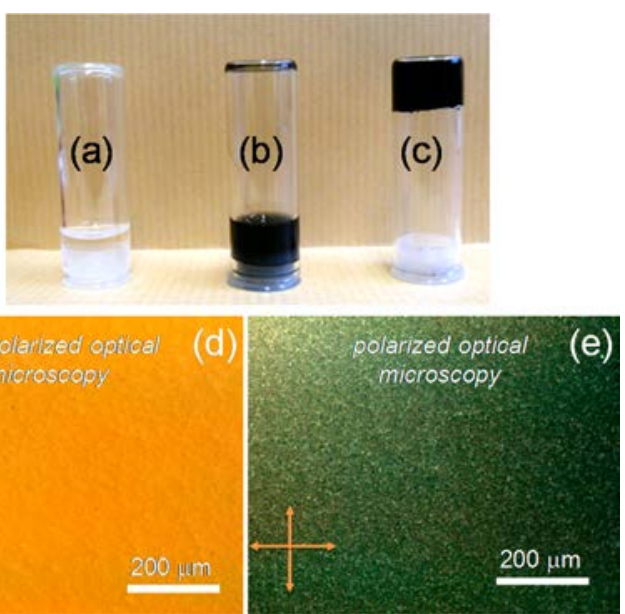

Figure 2. Macroscopic photographs of (a) a 0.5 wt \% HA aqueous solution; (b) an isotropic suspension with $0.3 \mathrm{wt} \%$ SWCNTs and 0.25 wt \% HA and (c) a HA-CNT biogel with 0.6 wt \% SWCNTs and 0.5 wt \% HA. Vials have been turned upside down to show the effect of gelation. Optical micrographs of a sample of the biogel with $0.6 \mathrm{wt} \%$ SWCNT and 0.5 wt \% of HA after one hour, after preparation and confinement in a $10 \mu \mathrm{m}$ thick quartz cell with (d) no polarization and (e) between crossed polarizers. Arrows indicate the orientations of the axes of the polarizers.

the presence of domains with oriented carbon nanotubes. These domains can be considered as domains of the nematic phase, which span the whole volume of the cell. A phase separation has actually taken place.

The size of the domains exceeds a micrometer, and it can therefore be expected that the kinetics of their formation and growth can be followed using SALS. A set of suspensions is therefore prepared with different amounts of SWCNTs in the range of $0.3-0.6 \mathrm{wt} \%$ and of HA in the range of $0.25-1 \mathrm{wt} \%$. Each sample is characterized by SALS, as function of its aging time $\left(t_{w}\right)$. Examples of scattering curves are shown in Figure 3 for a sample containing $0.4 \mathrm{wt} \%$ of SWCNT and $1 \mathrm{wt} \%$ of HA. The graph contains twenty curves, corresponding to successive measurements separated by $5 \mathrm{~min}$ regular intervals. The

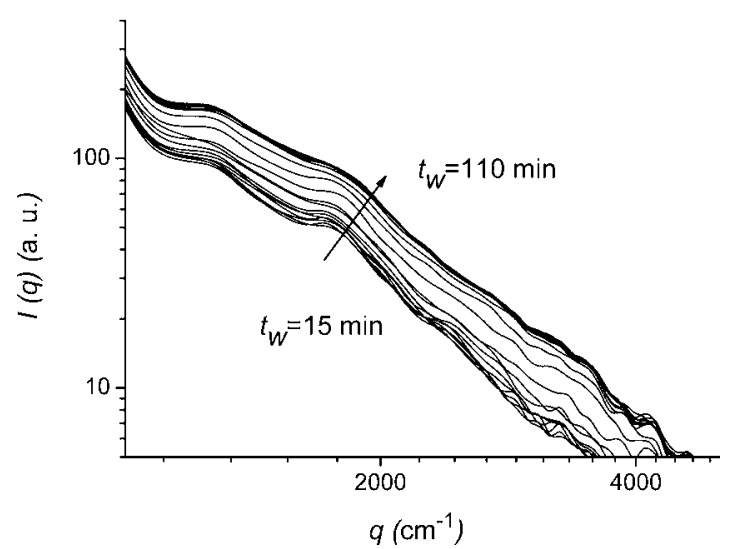

Figure 3. Intensity as a function of the wave vector $\mathrm{q}$ for different aging times $t_{w}$ for a sample containing $0.4 \mathrm{wt} \%$ of SWCNT and $1 \mathrm{wt} \%$ of HA. $t_{w}$ varies from 15 to $110 \mathrm{~min}$. The corresponding curves are separated by $5 \mathrm{~min}$ regular intervals between each measurement. It can be seen that the first lower curves and the last top curves are close to each other. This reflects the latency time before gelation and the absence of evolution at long times after gelation. 
scattered intensity increases when $t_{w}$ increases, from the bottom to the top of Figure 3. This reflects the formation of the nematic domains which scatter light at small angles. In order to more clearly follow the phase separation kinetics, we computed the average normalized scattered intensity in the range of 1355 $\mathrm{cm}^{-1}<q<4067 \mathrm{~cm}^{-1}$ for the different investigated samples. The normalized scattered intensity as a function of $t_{w}$ for three different samples with a constant SWCNT concentration of 0.4 wt $\%$ and different HA content, from 0.5 to 1 wt $\%$, is shown in Figure 4a. This figure also shows the same information for a
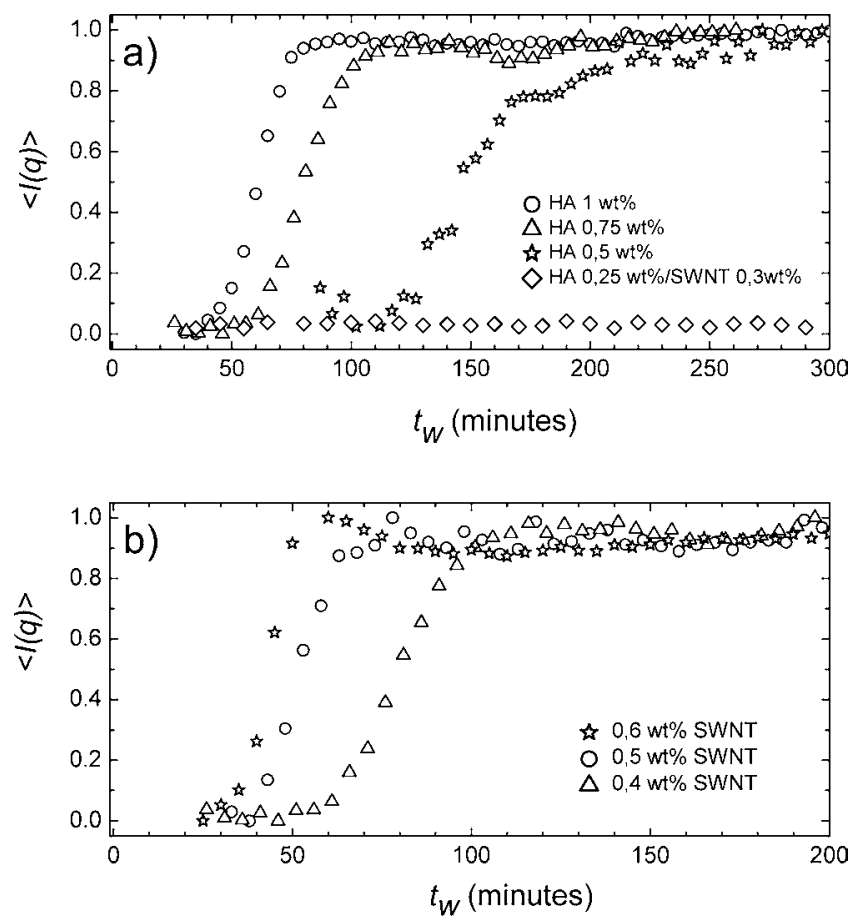

Figure 4. Normalized scattered intensity as a function of the aging time $\left(t_{w}\right)$ for (a) constant SWCNT concentration of $0.4 \mathrm{wt} \%$ and three different HA concentrations and (b) constant HA concentration of 0.75 wt $\%$ and three different SWCNT concentrations. In (a) the temporal evolution of the scattered light for an isotropic suspension with SWCNT 0.3 wt \%/HA 0.25 wt \% is also shown in (a).

slightly more diluted material that contains 0.3 wt \% of SWCNT and 0.25 wt \% of HA. The scattered intensity remains close to zero over the whole $q$ range for this more dilute system. By contrast, curves of concentrated samples exhibit a sigmoidal shape. This type of curve is typically observed in arrested phase separations. ${ }^{36}$ The intensity starts to increase after a certain latency times and then levels off at longer times, meaning that the system is dynamically frozen. The nematic domains do not coarsen, and the full phase separation cannot be achieved. Dynamical arrest in other colloid and macromolecular phase separations has been shown to arise from the effect of attractive interactions. ${ }^{34-39}$ The time to reach the arrested state or the time needed to form a gel can be defined as the inflection point in the curves shown in Figure 4. It is seen that the gelation time strongly decreases with increasing the amount of HA. Similar experiments were performed to show the effect of the SWCNT concentration. The normalized scattered intensity as a function of $t_{w}$ for three different samples, with a constant HA concentration of 0.75 wt $\%$ and different SWCNT content from 0.4 to $0.6 \mathrm{wt} \%$ is shown in Figure $4 \mathrm{~b}$. Again sigmoidal curves reflecting an arrested phase separation are obtained. The gelation time decreases significantly with increasing SWCNT concentration.

The gelation times for all samples investigated were determined as a function of the $\mathrm{HA}$ and SWCNT concentrations (Figure 5). It is clear that the gelation time

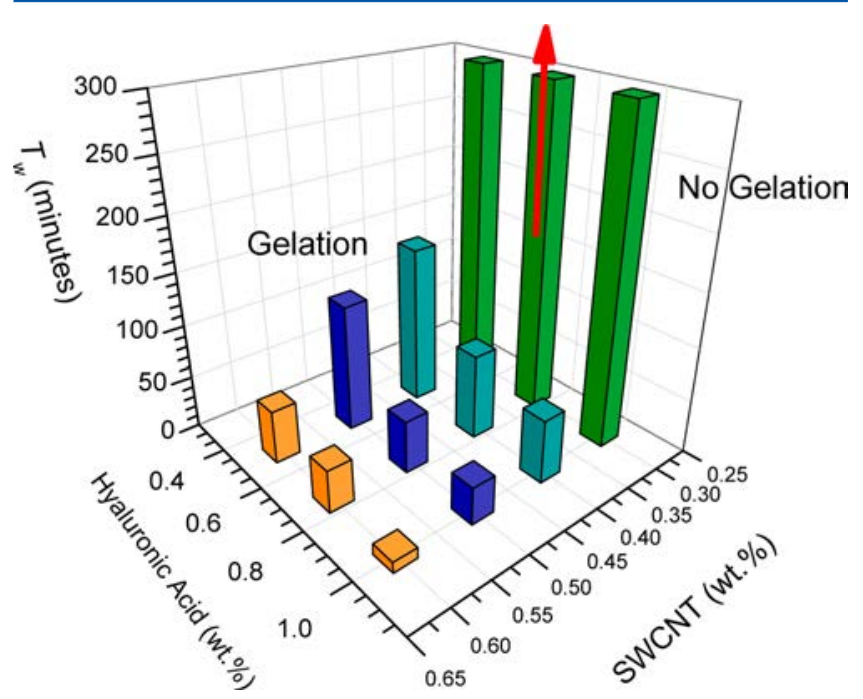

Figure 5. Gelation time of HA-SWCNT mixtures as a function of the weight fractions of HA and SWCNTs. For low concentration of SWCNT ( $0.3 \mathrm{wt} \%)$, the systems remain fluid; the gelation time would be considered as infinite as sketched by the red arrow pointing up.

decreases both with $\mathrm{HA}$ and nanotube contents. It is also observed that below certain SWCNT/HA concentrations the systems do not form gels. This is consistent with the fact that the gelation arises from a phase separation that takes place only above a given concentration of SWCNT/HA. The fact that the gelation is promoted by the addition of HA strongly supports that the HA molecules induce attractive interactions between the CNT and thereby promote the formation of a liquid crystal phase. $^{50}$ The latter has a sufficient elasticity to form a macroscopic gel. The elasticity of the nematic phase can arise from the presence of topological defects and entanglements of nanotubes which hinder the coarsening of the phase separation. ${ }^{51,52}$

Deeper insights on the gelation mechanisms can be obtained by investigating the spectroscopic and optical properties of the suspensions. Typical coupled PL/RS spectra are presented in Figure 6. All the spectra have been normalized by the G-band and shifted along the $y$ axis for clarity. In the bottom spectrum, we see the typical signature of HiPCO-SWCNTs bundles [i.e., Raman peaks corresponding to radial breathing modes (RBM) at low frequencies between 250 and $350 \mathrm{~cm}^{-1}, \mathrm{D}$ and $\mathrm{G}^{\prime}$ bands around 1280 and $260 \mathrm{~cm}^{-1}$, and $\mathrm{G}$ bunchs in the range of $1550-1600 \mathrm{~cm}^{-1}, 40,41$ superimposed to a weak and flat background. This spectrum was measured directly with the SWCNT wet-cake. By contrast, in the middle of Figure 6, we observe the Raman and the superimposed PL spectrum for a HA-SWCNTs isotropic suspension containing 0.3 wt \% SWCNT and 0.25 wt \% of HA. The Raman features are similar for nanotube bundles and suspensions, with RBM, D, G, and $G^{\prime}$ signatures at the same wave numbers. However, additional broad PL lines are observed in the spectra of suspensions. They reveal unambiguously the presence of individualized semiconducting nanotubes in the HA-SWCNTs dispersions. Note that HA molecules do not exhibit any PL. 


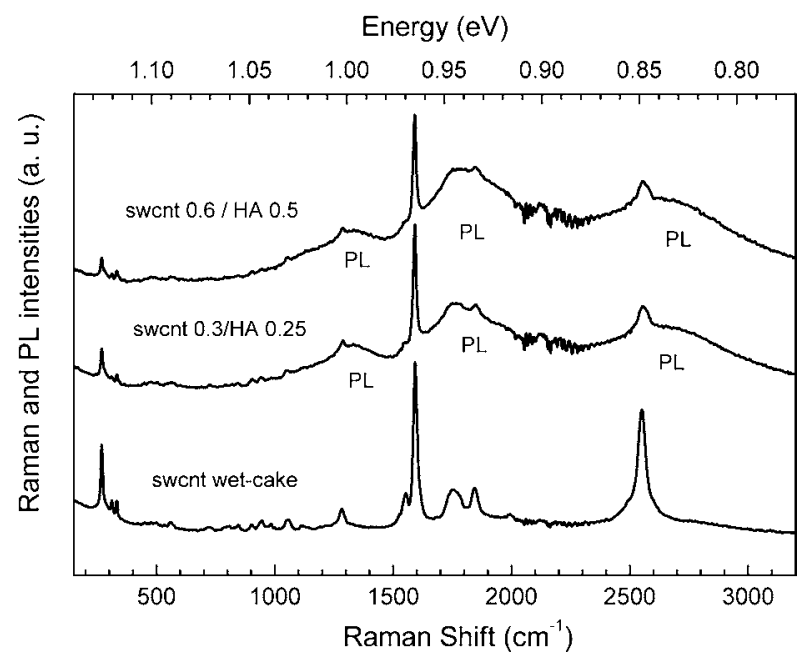

Figure 6. Coupled photoluminescence (energy on the top scale)/ Raman (Raman shift on the bottom scale) spectra obtained with an incident laser line of $1.16 \mathrm{eV}$ (from bottom to top): (i) purified SWCNT wet cake, (ii) SWCNT isotropic suspension with SWCNT 0.3 and 0.25 wt \% of HA, and (iii) SWCNT nematic gels with 0.6 wt \% of SWCNT and 0.5 wt \% of HA. PL peaks are indicated in the picture for suspensions and gels. Intensity fluctuations in the range of 2000$2400 \mathrm{~cm}^{-1}$ are due to the strong absorption bunch from atmospheric water vapor around $1380 \mathrm{~nm}$. Note also that the smallest relative intensity of the $\mathrm{G}^{\prime}$ band in suspensions and gels is due to the strong absorption of liquid water above $1300 \mathrm{~nm}$.

The present observations confirm the efficiency of HA molecules at being adsorbed at the CNT interface of the $\mathrm{CNT}$ and at stabilizing CNTs in water. In contrast to the case of other biomacromolecules, ${ }^{25}$ the observation of PL indicates that the HA molecules do not prevent unbundling of the nanotubes. More surprisingly, PL is also observed in gelled materials. The top curve in Figure 6 shows the Raman and PL spectrum after the liquid crystal phase transition has taken place for a sample containing 0.6 wt \% of SWCNT and $0.5 \mathrm{wt} \%$ of HA. Comparisons of this spectrum with that obtained for the isotropic suspension reveal that all the Raman and PL features are essentially unchanged (i.e., like Raman spikelike RBM, D, $\mathrm{G}$, and $\mathrm{G}^{\prime}$ bands at similar wavenumbers) and comparable PL broad lines at similar emission energies and intensities. These results confirm that nanotubes are individualized both in the isotropic phase and in the gelled phase. HA stabilized CNTs do not form bundles in response to strong van der Waals interactions. Instead, the CNTs are stabilized against close contact by short-range repulsive interactions. ${ }^{53}$ The latter can arise from steric and electrostatic interactions of the absorbed polyelectrolyte HA. Indeed, HA is a salt that is expected to be highly charged in solution as a consequence of the dissociation of potassium ions. In consideration of the molecular weight of a $\mathrm{HA}$ monomer and the concentrations of HA present in the investigated solutions, it can be deduced that the ionic strength is on the order of a few moles per liter. At such ionic strength, electrostatic interactions are expected to act at very short range. Actually, the Debye length which characterizes the range of the electrostatic repulsion is expected to be below $1 \mathrm{~nm}^{53}$ Electrostatic and steric repulsion act at short range and stabilize the CNTs against bundling. But these repulsions cannot balance attractive interactions at long range. Typically the range of depletion interaction induced by polymer in solutions is given by the radius of gyration of the polymer. In the present case, this means that attractive interactions are expected to act at distances above $10 \mathrm{~nm},{ }^{54}$ well above the range of electrostatic repulsions. This is why HA can play the role of a stabilizer at short range and still induce attractive interactions at long range. It is also interesting to note that the radius of gyration of the polymer can significantly vary with the molecular weight of the polymer. $^{54}$ Using sorted HA materials with a lower polydispersity could therefore allow a better control of the range of the depletion interactions and thereby a better control of the gel formation. In particular, it can be expected that the boundaries for gel formation should vary as a function of the radius of gyration. It is also important to note that the presently used carbon nanotubes are not oxidized and are therefore hydrophobic. The observed behaviors indicate that HA molecules strongly interact with hydrophobic interfaces. The observed phase behavior could significantly differ if the surface chemistry of the nanotubes would be modified. In particular, oxidized nanotubes would become hydrophilic because of the presence of hydroxyl and carboxylic groups. It is not obvious that HA molecules would efficiently adsorb on such hydrophilic and charged interfaces.

The strong PL intensity in all the investigated dispersions indicates that HA molecules wrap carbon nanotubes and remain strongly absorbed at their interface during and after gelation. These strong interactions explain the stability of HACNT biogels. Nevertheless, the presence of molecules at the interface of the CNT also provides a barrier for the electronic conduction of CNT assemblies. It is indeed known that the conductivity of CNT-based electrodes is often limited by the resistance of the junctions between nanotubes. It has been observed that HA-CNT electrodes surprisingly exhibit an impedance greater than that of composite electrodes made with other biopolymers, even at a large fraction of CNTs. ${ }^{15}$ The greater impedance of $\mathrm{HA}-\mathrm{CNT}$ electrodes could arise from the strong absorption of HA molecules. On the other hand, individualization of CNTs offer a significant advantage for bioimaging applications based on the near-infrared photoluminescence of the CNTs.

\section{CONCLUSION}

The combination of the present experimental observations allows some important conclusions to be drawn. The gelation of HA-CNT mixtures reflects an arrested phase separation arising from weak attractive interactions. The present interactions do not induce bundling of nanotubes and are likely to be depletion interactions due to the excess of polymer in solution. The time needed to form a gel depends strongly on the concentration of HA and CNTs. Nevertheless, the gelation time can be very short, of only a few minutes, even for relatively low concentrations of materials. This behavior demonstrates the remarkable efficiency of carbon nanotubes at forming HACNT gels. This exceptional efficiency can be understood by considering that the involved transition is a liquid crystal phase transition. Indeed carbon nanotubes exhibit a giant aspect ratio and are therefore prone to easily form liquid crystal phases at low concentrations. ${ }^{55}$ By contrast, spherical particles or particles with a small aspect ratio would not exhibit similar transitions at low concentration and are expected to be less efficient than carbon nanotubes at forming HA based gels. The present concepts therefore provide some guidance to optimize future CNT biogels. It can indeed be expected that CNT with a high aspect ratio would be more efficient at forming gels than short CNTs. We also believe that these concepts can be 
extended to other mixtures of biomacromolecules and rodlike particles and therefore offer a novel strategy for the design of functional and biocompatible biogels.

\section{AUTHOR INFORMATION}

\section{Corresponding Author}

*E-mail: poulin@crpp-bordeaux.cnrs.fr.

\section{Notes}

The authors declare no competing financial interest.

\section{ACKNOWLEDGMENTS}

We thank the European Commission for financial support of this work in the frame of the COMPLOIDS project. S.E.M. thanks the Australian Academy of Science for financial support in the form of the International Travel Award. G.G.W. and S.E.M. thank the Australian Research Council for their Australian Laureate Fellowship and Queen Elizabeth II Fellowship, respectively.

\section{REFERENCES}

(1) Kogan, G.; Soltes, L.; Stern, R.; Gemeiner, P. Hyaluronic acid: A natural biopolymer with a broad range of biomedical and industrial applications. Biotechnol. Lett. 2007, 29 (1), 17-25.

(2) Mori, M.; Yamaguchi, M.; Sumitomo, S.; Takai, Y. Hyaluronanbased biomaterials in tissue engineering. Acta Histochem. Cytochem. 2004, 37 (1), 1-5.

(3) Brown, M. B.; Jones, S. A. Hyaluronic acid: A unique topical vehicle for the localized delivery of drugs to the skin. J. Eur. Acad. Dermatol. Venereol. 2005, 19 (3), 308-318.

(4) Censi, R.; Di Martino, P.; Vermonden, T.; Hennink, W. E. Hydrogels for protein delivery in tissue engineering. J. Controlled Release 2012, 161 (2), 680-692.

(5) Kim, M. R.; Park, T. G. Temperature-responsive and degradable hyaluronic acid/Pluronic composite hydrogels for controlled release of human growth hormone. J. Controlled Release 2002, 80 (1-3), 69-77.

(6) Garcia-Abuin, A.; Gomez-Diaz, D.; Navaza, J. M.; Regueiro, L.; Vidal-Tato, I. Viscosimetric behaviour of hyaluronic acid in different aqueous solutions. Carbohydr. Polym. 2011, 85 (3), 500-505.

(7) Pisarcik, M.; Bakos, D.; Ceppan, M. Non-newtonian properties of hyaluronic-acid aqueous-solution. Colloids Surf., A 1995, 97 (3), 197202.

(8) Rinaudo, M. Non-covalent interactions in polysaccharide systems. Macromol. Biosci. 2006, 6 (8), 590-610.

(9) Shchipunov, Y. A.; Karpenko, T. Y.; Krekoten, A. V.; Postnova, I. V. Gelling of otherwise nongelable polysaccharides. J. Colloid Interface Sci. 2005, 287 (2), 373-378.

(10) Collins, M. N.; Birkinshaw, C. Physical properties of crosslinked hyaluronic acid hydrogels. J. Mater. Sci.: Mater. Med. 2008, 19 (11), $3335-3343$.

(11) Dhanasingh, A.; Salber, J.; Moeller, M.; Groll, J. Tailored hyaluronic acid hydrogels through hydrophilic prepolymer crosslinkers. Soft Matter 2010, 6 (3), 618-629.

(12) Schmut, O.; Hofmann, H. Preparation of gels from hyaluronate solutions. Graefes Archive for Clinical and Experimental Ophthalmology 1982, 218 (6), 311-314.

(13) Moulton, S. E.; Maugey, M.; Poulin, P.; Wallace, G. G. Liquid crystal behavior of single-walled carbon nanotubes dispersed in biological hyaluronic acid solutions. J. Am. Chem. Soc. 2007, 129 (30), 9452-9457.

(14) Bhattacharyya, S.; Guillott, S.; Dabboue, H.; Tranchant, J. F.; Salvetat, J. P. Carbon nanotubes as structural nanofibers for hyaluronic acid hydrogel scaffolds. Biomacromolecules 2008, 9 (2), 505-509.

(15) Thompson, B. C.; Moulton, S. E.; Gilmore, K. J.; Higgins, M. J.; Whitten, P. G.; Wallace, G. G. Carbon nanotube biogels. Carbon 2009, 47 (5), 1282-1291.

(16) Filip, J.; Sefcovicova, J.; Tomcik, P.; Gemeiner, P.; Tkac, J. A hyaluronic acid dispersed carbon nanotube electrode used for a mediatorless NADH sensing and biosensing. Talanta 2011, 84 (2), 355-361.

(17) Lynam, C.; Moulton, S. E.; Wallace, G. G. Carbon-nanotube biofibers. Adv. Mater. 2007, 19 (9), 1244-1248.

(18) Pelto, J.; Haimi, S.; Puukilainen, E.; Whitten, P. G.; Spinks, G. M.; Bahrami-Samani, M.; Ritala, M.; Vuorinen, T. Electroactivity and biocompatibility of polypyrrole-hyaluronic acid multi-walled carbon nanotube composite. J. Biomed. Mater. Res., Part A 2010, 93A (3), 1056-1067.

(19) Shangguan, X. D.; Zheng, J. B.; Zhang, H. F.; Tang, H. S. Direct electrochemistry and electrocatalysis behaviors of glucose oxidase based on hyaluronic acid-carbon nanotubes-ionic liquid composite film. Chin. J. Chem. 2010, 28 (10), 1890-1896.

(20) Wang, Q.; Zheng, J. B. Direct electrochemistry and electrocatalysis of horseradish peroxidase immobilized in hyaluronic acid and single walled carbon nanotubes composite film. Chem. Pap. 2010, 64 (5), 566-572.

(21) Xing, X. R.; Liu, S.; Yu, J. H.; Lian, W. J.; Huang, J. D. Electrochemical sensor based on molecularly imprinted film at polypyrrole-sulfonated graphene/hyaluronic acid-multiwalled carbon nanotubes modified electrode for determination of tryptamine. Biosens. Bioelectron. 2012, 31 (1), 277-283.

(22) Asakura, S.; Oosawa, F. On interaction between 2 bodies immersed in a solution of macromolecules. J. Chem. Phys. 1954, 22 (7), 1255-1256.

(23) Vigolo, B.; Coulon, C.; Maugey, M.; Zakri, C.; Poulin, P. An experimental approach to the percolation of sticky nanotubes. Science 2005, 309 (5736), 920-923.

(24) Bomboi, F.; Bonincontro, A.; La Mesa, C.; Tardani, F. Interactions between single-walled carbon nanotubes and lysozyme. J. Colloid Interface Sci. 2011, 355 (2), 342-347.

(25) Edri, E.; Regev, O. "Shaken, Not Stable": Dispersion mechanism and dynamics of protein-dispersed nanotubes studied via spectroscopy. Langmuir 2009, 25 (18), 10459-10465.

(26) Duggal, R.; Pasquali, M. Dynamics of individual single-walled carbon nanotubes in water by real-time visualization. Phys. Rev. Lett. 2006, 96, 24.

(27) Fakhri, N.; Tsyboulski, D. A.; Cognet, L.; Weisman, R. B.; Pasquali, M. Diameter-dependent bending dynamics of single-walled carbon nanotubes in liquids. Proc. Ntl. Acad. Sci. U.S.A. 2009, 106 (34), 14219-14223.

(28) Yakobson, B. I.; Couchman, L. S. Persistence length and nanomechanics of random bundles of nanotubes. J. Nanopart. Res. 2006, 8 (1), 105-110.

(29) Carpineti, M.; Giglio, M. Spinodal-type dynamics in fractal aggregation of colloidal clusters. Phys. Rev. Lett. 1992, 68 (22), 33273330.

(30) Bibette, J.; Mason, T. G.; Hu, G.; Weitz, D. A.; Poulin, P. Structure of adhesive emulsions. Langmuir 1993, 9 (12), 3352-3356.

(31) Poulin, P.; Bibette, J.; Weitz, D. A. From colloidal aggregation to spinodal decomposition in sticky emulsions. Eur. Phys. J. B 1999, 7 (2), $277-281$.

(32) Cipelletti, L.; Manley, S.; Ball, R. C.; Weitz, D. A. Universal aging features in the restructuring of fractal colloidal gels. Phys. Rev. Lett. 2000, 84 (10), 2275-2278.

(33) Cipelletti, L.; Weitz, D. A. Ultralow-angle dynamic light scattering with a charge coupled device camera based multispeckle, multitau correlator. Rev. Sci. Instrum. 1999, 70 (8), 3214-3221.

(34) Lu, P. J.; Zaccarelli, E.; Ciulla, F.; Schofield, A. B.; Sciortino, F.; Weitz, D. A. Gelation of particles with short-range attraction. Nature 2008, 453 (7194), 499-U4.

(35) Cardinaux, F.; Gibaud, T.; Stradner, A.; Schurtenberger, P. Interplay between spinodal decomposition and glass formation in proteins exhibiting short-range attractions. Phys. Rev. Lett. 2007, 99 (11).

(36) Foffi, G.; De Michele, C.; Sciortino, F.; Tartaglia, P. Arrested phase separation in a short-ranged attractive colloidal system: A numerical study. J. Chem. Phys. 2005, 122 (22), 224903. 
(37) Puertas, A. M.; Fuchs, M.; Cates, M. E. Competition between glass transition and liquid-gas separation in attracting colloids. J. Phys.: Condens. Matter 2007, 19, 20.

(38) Trappe, V.; Prasad, V.; Cipelletti, L.; Segre, P. N.; Weitz, D. A. Jamming phase diagram for attractive particles. Nature 2001, 411 (6839), 772-775.

(39) Zaccarelli, E.; Lu, P. J.; Ciulla, F.; Weitz, D. A.; Sciortino, F. Gelation as arrested phase separation in short-ranged attractive colloid-polymer mixtures. J. Phys.: Condens. Matter 2008, 20 (49).

(40) Dresselhaus, M. S.; Dresselhaus, G.; Saito, R.; Jorio, A. Raman spectroscopy of carbon nanotubes. Phys. Rep. 2005, 409 (2), 47-99.

(41) Reich, S.; Thomsen, C.; Maultzsch, J.. Carbon Nanotubes: Basic Concepts and Physical Properties: Wiley-VCH, 2004.

(42) O'Connell, M. J.; Bachilo, S. M.; Huffman, C. B.; Moore, V. C.; Strano, M. S.; Haroz, E. H.; Rialon, K. L.; Boul, P. J.; Noon, W. H.; Kittrell, C.; Ma, J. P.; Hauge, R. H.; Weisman, R. B.; Smalley, R. E. Band gap fluorescence from individual single-walled carbon nanotubes. Science 2002, 297 (5581), 593-596.

(43) Nikolaev, P.; Bronikowski, M. J.; Bradley, R. K.; Rohmund, F.; Colbert, D. T.; Smith, K. A.; Smalley, R. E. Gas-phase catalytic growth of single-walled carbon nanotubes from carbon monoxide. Chem. Phys. Lett. 1999, 313 (1-2), 91-97.

(44) Zamora-Ledezma, C.; Anez, L.; Primera, J.; Silva, P.; EtienneCalas, S.; Anglaret, E. Photoluminescent single wall carbon nanotubesilica composite gels. Carbon 2008, 46 (9), 1253-1255.

(45) Chiang, I. W.; Brinson, B. E.; Huang, A. Y.; Willis, P. A.; Bronikowski, M. J.; Margrave, J. L.; Smalley, R. E.; Hauge, R. H. Purification and characterization of single-wall carbon nanotubes obtained from the gas-phase decomposition of $\mathrm{CO}$ (HiPco process). J. Phys. Chem. B 2001, 105 (35), 8297-8301.

(46) Badaire, S.; Poulin, P.; Maugey, M.; Zakri, C. In situ measurements of nanotube dimensions in suspensions by depolarized dynamic light scattering. Langmuir 2004, 20 (24), 10367-10370.

(47) Lucas, A.; Zakri, C.; Maugey, M.; Pasquali, M.; van der Schoot, P.; Poulin, P. Kinetics of Nanotube and Microfiber Scission under Sonication. J. Phys. Chem. C 2009, 113 (48), 20599-20605.

(48) Pagani, G.; Green, M. J.; Poulin, P.; Pasquali, M. Competing mechanisms and scaling laws for carbon nanotube scission by ultrasonication. Proc. Natl. Acad. Sci. U.S.A. 2012, 109 (29), 1159911604 .

(49) Ferri, F. Use of a charge coupled device camera for low-angle elastic light scattering. Rev. Sci. Instrum. 1997, 68 (6), 2265-2274.

(50) Lekkerkerker, H. N. W.; Stroobants, A. Phase-behavior of rodlike colloid plus flexible polymer mixtures. Nuovo Cimento Soc. Ital. Di Fis., $D$ 1994, 16, 949-962.

(51) Puech, N.; Blanc, C.; Grelet, E.; Zamora-Ledezma, C.; Maugey, M.; Zakri, C.; Anglaret, E.; Poulin, P. Highly ordered carbon nanotube nematic liquid crystals. J. Phys. Chem. C 2011, 115, 3272-3278.

(52) Zamora-Ledezma, C.; Blanc, C.; Maugey, M.; Zakri, C.; Poulin, P.; Anglaret, E. Anisotropic thin films of single-wall carbon nanotubes from aligned lyotropic nematic suspensions. Nano Lett. 2008, 8, 41034107.

(53) Hunter, R. J. Foundation of Colloid Science, 2nd ed.; Oxford University Press: Oxford, U.K., 2001.

(54) Mendichi, R.; Soltes, L.; Schieroni, A. G. Evaluation of radius of gyration and intrinsic viscosity molar mass dependence and stiffness of hyaluronan. Biomacromolecules 2003, 4 (6), 1805-1810.

(55) Onsager, L. The effects of shape on the interaction of colloidal particles. Ann. N.Y. Acad. Sci. 1949, 51 (4), 627-659. 